\title{
The effect of Angelica dahurica extract on breast carcinogenesis in mouse
}

\author{
Ruihong Zhang, Teng Liu, Xiangli Zhang, Yuan Liu
}

Obstetric Department, Qilu Hospital, Shandong University, China

Submitted: 17 March 2020

Accepted: 25 June 2020

Arch Med Sci

DOI: https://doi.org/10.5114/aoms.2020.101281

Copyright $\odot 2020$ Termedia \& Banach

\begin{abstract}
Introduction: Angelica dahurica extract (ADE) has been demonstrated to possess functions of immune response modulation, breast injury and fibrosis amelioration, breast regeneration regulation, and suppression of breast cancer.

Material and methods: To explore the effect of the ADE on breast cancer generation and epithelial-mesenchymal transition (EMT) in vivo, a breast cancer mouse model was established. Chinese traditional medicine ADE was administered to mice bearing breast cancer, which were randomly divided into a negative group, ADE group (two doses), and model group. The breast tissue of the mouse model was fixed and H\&E staining was performed for subsequent histological observation. Immunofluorescence assay and immunohistochemical staining were performed to examine the breast cancer related proteins, SNCG and BCRP in breast tissue; Western blotting and quantitative real-time polymerase chain reaction (PCR) were performed to detect proteins and gene expression of epithelial-mesenchymal transition (EMT)-related markers, E-cadherin, $\mathrm{N}$-cadherin, vimentin, transforming growth factor $\beta$ (TGF- $\beta$ ), and SNAIL, as well as signals in the MAPK and JAK/ STAT signaling pathways.

Results: Pathological manifestation of breast tissues in ADE-treated mouse was clearly reduced in both visual inspection and H\&E staining. Breast cancer specific SNCG and BCRP expression significantly decreased after ADE administration. Furthermore, increased $\mathrm{E}$-cadherin and decreased $\mathrm{N}$-cadherin, vimentin, SNAIL and TGF- $\beta$ expression might be associated with the antiEMT property of ADE. Both MAPK and JAK/STAT signaling pathways were observed to be inactivated after ADE treatment.

Conclusions: Collectively, the evidence showed that ADE restrained breast cancer development in vivo through blocking the EMT process, probably by down-regulation of MAPK and JAK/STAT signaling pathways.
\end{abstract}

Key words: Angelica dahurica extract, breast cancer, EMT, mouse model.

\section{Introduction}

Breast cancer has been widely reported as the second cause of deaths in women worldwide [1-3], while metastasis of tumor cells is the major cause of breast cancer related deaths $[2,4,5]$. Most of the breast cancer patients died of metastasis, rather than the primary tumors, and the 5 -year survival rate is a mere $23 \%$ for the women diagnosed with distant metastatic disease $[3,6]$. However, its pathogenesis is still unclear and there is a lack of effective therapy.

\author{
Corresponding author: \\ Prof. Yuan Liu \\ Obstetric Department \\ Qilu Hospital \\ Shandong University, China \\ E-mail: \\ liuyuanqilu54321@163.com
}


Epithelial-mesenchymal transition (EMT) is a recent hotspot in carcinoma metastasis research. In the beginning, EMT is widely considered to be a critical developmental program that transfers epithelial cells to mesenchymal cells, mobilizing cells and giving rise to bone, muscle, blood vessels, and connective tissue [7]. Increasing research has shown that the EMT program is implicated in cancer progression and metastasis including carcinogenesis, increased mobility, invasion, and angiogenesis in various solid tumor types [8]. The changes during EMT processes promote a shift in cytoskeletal dynamics and epithelial to mesenchymal morphology alteration [9]. In addition, several oncogenic transduction pathways that are responsible for extracellular signals have been shown to contribute to EMT, such as JAK/STAT, transforming growth factor $\beta$ (TGF- $\beta$ ), Wnt/ $\beta$-catenin, Notch, EGFR and MAPK signaling pathways [10]. These pathways have activated transcription factors including E-cadherin, $\mathrm{N}$-cadherin, vimentin, SNAIL, and TGF- $\beta$, to generate many intermediate characteristics from epithelial to mesenchymal cells [11].

Recently, Chinese traditional medicine (CTM) and natural compounds (derivative) obtained from a variety of herbs have become popular as alternative and complementary therapies [12]. CTM provides alternative anti-neoplastic, anti-inflammatory, and anti-microbial therapies [13]. Angelica dahurica extract (ADE) possesses anti-neoplastic activity in different kinds of cancer [14-17]. In the present study, ADE was applied in a breast tumor mouse model to investigate its anti-tumor effects in vitro.

Several reports have indicated that EMT was involved in tumor initiation and metastasis in vivo [18-22], and differentiated into malignant cells in vitro [23-26]. In addition, EMT is one of the essential mechanisms of breast tumorigenesis [27, 28]. Therefore, in the present study, we focused on the period of cancer cell metastasis and EMT in the established breast cancer mouse model, and observed the therapeutic effect of CTM ADE on breast tumorigenesis, metastasis, and the EMT process, and eventually attempted to reveal the mechanism of breast carcinoma generation.

\section{Material and methods}

\section{Experimental animal and cells}

Forty 5-6-week female SPF BALB/c nude mice were provided by the Animal Centre of Shandong University Centers for Disease Control. MDAMB-231 cells were purchased from the Chinese Academy of Medical Sciences (Beijing, China). The cells were cultured with DMEM culture medium (GIBCO-BRL, Grand Island, NY), with 10\% FBS (GIBCO-BRL) as a supplement under $5 \% \mathrm{CO}_{2}$ at $37^{\circ} \mathrm{C}$.

\section{Reagents and antibodies}

TRIzol reagent was purchased from Invitrogen; ReverTra Ace qPCR RT Kit and Thunderbird SYBR qPCR Mix were obtained from Toyobo; SABC Immunohistochemistry Kit was from Wuhan Boshide Biotech; Rabbit/mouse anti-human BCRP, SNCG, E-cadherin, N-cadherin, vimentin, SNAIL, and TGF- $\beta 1$ antibody, and rabbit/mouse anti-human MEK1, ERK1, VEGF, JAK2, STAT3, and STAT5 antibody were purchased from Abcam.

\section{Preparation of ADE}

Angelica dahurica powder (Sichuan, China) used was purchased from TongRenTang Biopharm Co., Ltd. Beijing City. Angelica dahurica powder (50 g) was boiled in $500 \mathrm{ml}$ of $70 \%$ ethanol for $1.5 \mathrm{~h}$ and the residue was boiled likewise again. The ethanol extract of Angelica dahurica Radix was collected and centrifuged at $12,000 \times \mathrm{g}$ for $15 \mathrm{~min}$ at $18^{\circ} \mathrm{C}$. The supernatant was condensed to $45 \mathrm{ml}$ in a rotary evaporator system (R-210, BUCHI, Switzerland) at $120 \mathrm{mbar}$ pressure at $60^{\circ} \mathrm{C}$. The condensed solution was partitioned with $50 \mathrm{ml}$ of ethyl acetate twice to yield the ethyl acetate ADE. The extract was dried by lyophilization overnight. The extract of $\mathrm{ADE}$ was stored at $-20^{\circ} \mathrm{C}$ prior to use.

Then, HPLC analysis of the ADE fraction was conducted according to a previous method [29]. The mixed standard solution for imperatorin and isoimperatorin (Sigma-Aldrich Inc., St. Louis, MO, USA) was prepared at a concentration of $0.1 \mathrm{mg} /$ $\mathrm{ml}$ (Acetonitrile: DMSO = $1: 1, \mathrm{v} / \mathrm{v}$ ). Likewise, $5 \mathrm{mg}$ of extract was prepared at the same concentration. The standard solution and the extract were purified by $0.22 \mu \mathrm{m}$ polypropylene filter and injected into an HP1100 series HPLC system equipped with a diode-array detector at $254 \mathrm{~nm}$ with an analytical HPLC column (ALLTIMA C18, $5 \mu \mathrm{m}, 250 \times 4.6 \mathrm{~mm}$ i.d.). The flow rate of the elution profile was set at $0.75 \mathrm{ml} / \mathrm{min}$. The gradient mobile phase was composed of solvent A $(0.1 \%$ trifluoroacetic acid) and solvent B (acetonitrile). The gradient for separation was programmed as follows: $0 \mathrm{~min}, 2 \% \mathrm{~B} ; 10 \mathrm{~min}, 5 \% \mathrm{~B} ; 15 \mathrm{~min}, 20 \%$ B; $35 \mathrm{~min}, 40 \% \mathrm{~B} ; 40 \mathrm{~min}, 60 \% \mathrm{~B} ; 70 \mathrm{~min}, 70 \%$ B; $80 \mathrm{~min}, 95 \% \mathrm{~B} ; 90 \mathrm{~min}, 100 \% \mathrm{~B}$ and held for an additional $5 \mathrm{~min}$. Calibration curves were peak area versus concentration for each standard solution. Quantification was performed upon six levels of external standards. The limit of quantification (LOQ) was determined as the concentration with a signal-to-noise ratio of ten.

\section{Establishment of breast cancer mouse model}

To assess the effect and mechanism of ADE on breast tumorigenesis and development in vivo, a xe- 
nograft mouse model with breast cancer was established [30]. Human breast cancer MDA-MB-231 cells $\left(2 \times 10^{5}\right.$ per mouse) were subcutaneously injected into nude mice. After cell inoculation, the mice were randomly divided into three groups $(n=6)$ : ADE (250 and $25 \mathrm{mg} / \mathrm{kg} /$ week) treatment groups (high dose ADE (hADE) and low dose ADE (IADE)), and a normal saline (NS) treatment group (model). The non-inoculation group was set as a negative control group (NC). Four weeks after tumor cell injection, 25 or $250 \mathrm{mg} / \mathrm{kg}$ ADE was administered by gavage on a regimen for 15 days post-injection for the lowand high-dose group. NS was given in the model group. To detect the tumorigenesis and metastasis, the mice were sacrificed 8 weeks after inoculation. For tumorigenesis observation, tumor growth was assessed by measuring the length and width of tumors with electronic calipers every 3-4 days continuously. Volumes were calculated using the formula: (length) $\times$ (width) $2 / 2$. For metastasis observation, their lungs were collected and sectioned with hematoxylin and eosin (H\&E) staining.

\section{Animal ethics statement}

Female BALB/c mice were purchased from $\mathrm{Vi}$ tal River Laboratories (Beijing, China) to establish a breast orthotopic xenograft tumor model. All animal studies were strictly conducted in accordance with the requirements of the animal experimental protocol, which was approved by the Animal Ethics Committees of Shandong University.

\section{H\&E staining and immunohistochemical (IHC) staining}

$H \& E$ staining was done using 5 - $\mu$ m-thick formalin-fixed, paraffin-embedded tissue sections. Tumor specimens from lung metastatic nodules of mice were fixed in $4 \%$ paraformaldehyde and then embedded in paraffin. Paraffin blocks were cut into 5-mm-thick sections. After washing with ethyl alcohol, the sections were dyed with hematoxylin solution for $3 \mathrm{~min}$, followed by incubation with eosin solution staining for $3 \mathrm{~min}$. The specimens were observed using a Zeiss Axioscope light microscope ( $\times 200$, Zeiss). The randomly selected slides in each group were magnified 400 -fold, and a semi-quantitative scoring method was used to evaluate the pathological degree of liver cancer. Edmondson-Steiner grading and scoring on positive cells was used to analyze the average pathological degree of mouse breast in different groups.

The expression of SNCG and BCRP was evaluated with IHC staining of 4- $\mu \mathrm{m}$-thick sections from the tissue blocks. The sections were first deparaffinized in xylene and then rehydrated through graded ethanol washes. For antigen retrieval, we performed autoclave heating of the sections at $100^{\circ} \mathrm{C}$ for $30 \mathrm{~min}$ in a sodium citrate buffer $(\mathrm{pH}$ 6.0). Endogenous peroxidase activity was blocked with peroxidase blocking solution (S2023; DakoCytomation, Carpinteria, USA). Slides were incubated with primary antibodies at $4^{\circ} \mathrm{C}$ overnight and incubated with labeled polymer (DAKO REAL EnVision/HRP, K5007; DakoCytomation) for 30 min at room temperature. The primary antibodies were rabbit anti-mouse SNCG (ab55424, Abcam) and rabbit anti-mouse BCRP (106937-T08, Sino Biological) polyclonal antibodies used at a $1: 1000$ dilution. 3,3'-Diaminobenzidine was used as a chromogen for detection, and Mayer's hematoxylin counterstain was applied.

\section{Matrigel invasion assay}

Matrigel (Thermo) was used to coat the upper segment of the Transwell chamber with polycarbonate membrane (Millipore) of $10 \mathrm{~mm}$ diameter and $8 \mu \mathrm{m}$ pore size overnight. MDA-MB-231 cells were starved for $12 \mathrm{~h}$ and loaded onto the upper segment $\left(5 \times 10^{5} /\right.$ well $)$ alone or in combination with varying concentrations of ADE $(50 \mathrm{nM}$, $100 \mathrm{nM}$ or $200 \mathrm{nM})$. The lower chambers were supplemented with RPMI-1640 medium (Thermo) with additional $1 \%$ FBS. Following incubation for $12 \mathrm{~h}, 5 \%$ polytetrafluoroethylene (PFA) and crystal violet were used to fix and stain the cells migrating into the lower chambers. Results were assessed with fluorescent microscopy. The experiments were performed in triplicate.

\section{Immunofluorescence assays}

Immunofluorescence assays (IFAs) were performed with a Leica SP8 confocal laser scanning microscope used for the IFA observation. PBST (PBS containing 1\% Triton X-100) and 5\% paraformaldehyde was used to rinse and fix the breast cancer cells on the coverslips. Coverslips were then incubated with anti-SNCG and BCRP antibodies in 5\% bovine serum albumin (BSA in PBST). Secondary antibodies conjugated with FITC and rhodamine were used to incubate with cells. The cellular nucleus was stained with DAPI.

\section{Western blotting (WB)}

Breast cancer tissue was lysed using RIPA lysis buffer (Sijiqing, Hangzhou, China). After incubation for $60 \mathrm{~min}$ at $4^{\circ} \mathrm{C}$, cell debris was removed by high speed centrifugation for $20 \mathrm{~min}$. $50 \mu \mathrm{g}$ of each sample were separated by SDS-PAGE (10\%) and transferred to PVDF membranes. The membranes were incubated with anti-E-cadherin antibody (1 : 500, ab76055, Abcam), anti-N-cadherin antibody (1 : 500, ab76057, Abcam), anti-Vimentin antibody (1 : 1000, ab8979, Abcam), anti-SNAIL antibody ( 1 : 500, ab53519, Abcam), anti-TGF- $\beta 1$ 
antibody (1 : 1000, ab92486, Abcam), and anti- $\beta$-actin antibody (1: 5000, ab8227, Abcam), followed by an HRP-conjugated anti-rabbit IgG secondary antibody (no.: BA1055, 1 : 2,500; Boster). The samples were visualized by ECL chemiluminescence. $\beta$-actin was used as an endogenous control.

\section{Quantitative real-time PCR (qRT-PCR)}

Total whole cell RNA of breast cancer cells was extracted using $1 \mathrm{ml}$ of TRIzol. Random hexamers was used to reverse-transcribe the RNA $(10 \mu \mathrm{g})$ from each group by PCR. The specific primers and GAPDH primers used are as follows: E-cadherin, F: GGG TTG TCT CAG CCA ATG TT, R: CAC CAA CAC ACC CAG CAT AG; Vimentin, F: AGA TCG ATG TGG ACG TTT CC, R: CAC CTG TCT CCG GTA TTC GT; Raf, F: AGC AAT GGT TTC GGA CTC AA, R: GCT TTC ATA AGG CAG TCG TG; MEK1, F: GTC CTA CAT GTC GCC TGA GA, R: GAG GTC GGC TAT CCA TTC CA; ERK1, F: GGC TTT CTG ACC GAG TAT GTG, R: TTT AGG TCC TCT TGG GAT GG; JAK2, F: CCC TGG CTG TCT ATA ACT CC, R: TCT GTA CCT TAT CCG CTT CC; STAT3 F: TCA CTT GGG TGG AAA AGG AC, R: TGG GAA TGT CAG GGT AGA GG; GAPDH F: TGT TGC CAT CAA CGA CCC CTT, R: CTC CAC GAC ATA CTC AGC A.

A control reaction was performed with no reverse transcriptase addition. $A B \mid 7300$ was used to analyze the relative specific gene expression, performed using PCR primers. $\mathrm{PPCR}$ was carried out using SYBR green. The cycling conditions comprised an initial denaturation step of $30 \mathrm{~s}$ at $95^{\circ} \mathrm{C}$, followed by 40 two-step cycles $\left(95^{\circ} \mathrm{C}\right.$ for $1-15 \mathrm{~s}$ and $62^{\circ} \mathrm{C}$ for $5-30 \mathrm{~s}$ ). Dissociation curve analysis was performed after each assay to ensure specific target detection.

\section{Colony generation assay}

Cells were resuspended 2 days following transfection in DMEM supplemented with 10\% FBS and $8 \mathrm{~mm} 0.4 \%$ top agar prior to being transferred to 12 -well plates containing $0.5 \mathrm{ml} 0.5 \%$ bottom agar. After 14 days, three regions were chosen randomly in each plate and colonies were quantified.

\section{Statistical analysis}

For all data SPSS 19.0 software was used for statistical analysis. The results are presented as mean \pm SD (mean \pm standard deviation). Multiple groups were compared using ANOVA analysis and a $p$ value $<0.05$ was considered statistically significant.

\section{Results}

\section{Visual inspection of breast cancer}

According to the previous investigation by the Gilles group [31], MDA-MB-231 is a well-laborato- ry adopted EMT-inducible model. To study whether ADE regulates the tumorigenesis, metastasis, and EMT process, an orthotopic xenograft mouse model was established by inoculating MDAMB-231 cells in the mammary fat pad of BALB/C nude mice. It was found that compared with the NC group, the breast tissue in the model group showed an irregular shape with different sizes on the surface or section. Various degrees of bleeding and necrosis were observed. These pathological manifestations were found to be reduced significantly after hADE treatment, whereas IADE treatment did not display a difference from the model group. Breast morphology in the NC group and hADE group showed a smooth surface, clear-cut margin, and soft texture. We observed that high dose use of ADE significantly relieved the body weight increment caused by breast tumor proliferation (Figure $1 \mathrm{~A}$ ). Furthermore, the tumor weight data showed that ADE treatment markedly reduced the tumor weight and tumor volume, compared with the model group (Figures $1 \mathrm{~B}$ and C).

\section{Effect of ADE on the pathologic degree of breast cancer}

To reveal the effect of ADE on pathologic degree of breast cancer, H\&E staining was performed in the mouse breast. It was found that tumor cells grew exuberantly in a higher density and disorganized manner in the model group and IADE group (Figure 2). These pathological manifestations were alleviated after being treated with hADE. The tissue in the NC group showed normal and arranged breast cell structure.

\section{Effect of ADE on breast tumor-relative protein expression}

Immunohistochemical staining was performed to detect the tumor-related protein, breast cancer cell specific marker SNCG (synuclein- $\gamma$ ) and BCRP (breast cancer relative protein) expression in each group. The SNCG and BCRP protein expression in the hADE group $(0.2423 \pm 0.0144$ and 0.4787 \pm 0.0228 , respectively) and these values in the IADE group (0.2514 \pm 0.0203 and $0.4790 \pm 0.0274$, respectively) were statistically significantly decreased comparing with these values in the model group $(0.3276 \pm 0.0210$ and $0.5887 \pm 0.0361$, respectively) ( $p<0.05$ ). Additionally, the expression pattern between the ADE group and NC group did not show a significant difference $(p>0.05)$ (Figure $3 \mathrm{~A})$. IFAs were performed to detect the co-expression of SNCG, BCRP, CD34, and CD45 in breast tissue cells in each group. Red fluorescence coupled with SNCG and BCRP expression while green fluorescence coupled with CD34 and CD45 expression. Yellow/orange fluorescence, representing the 
A

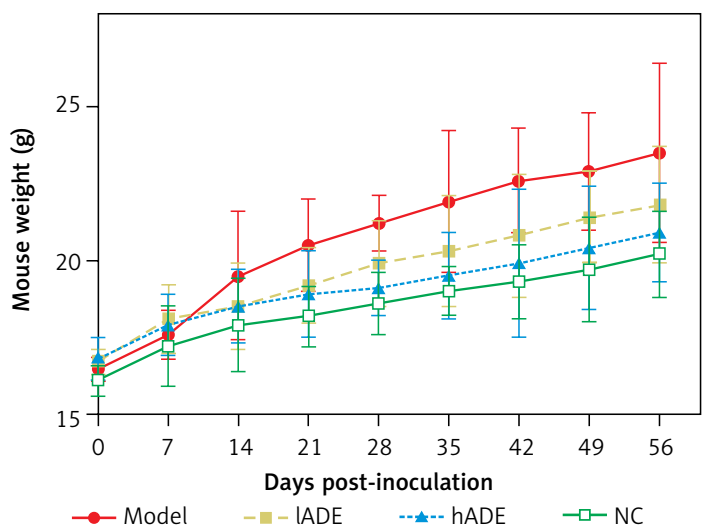

B

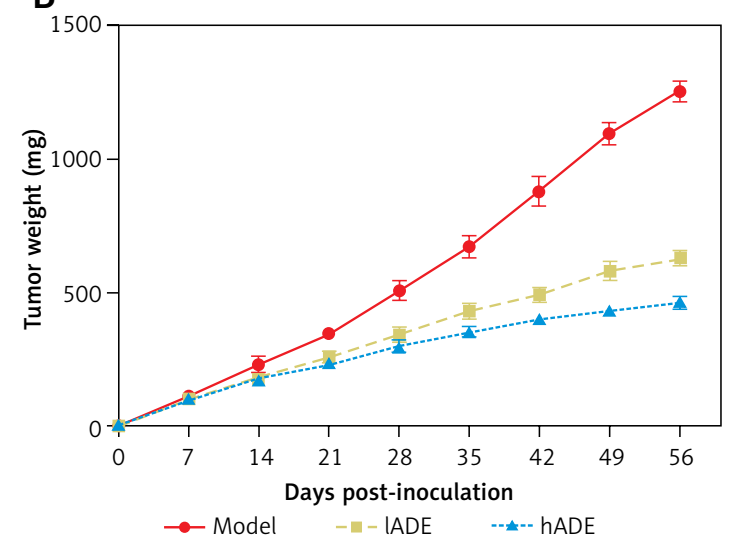

C

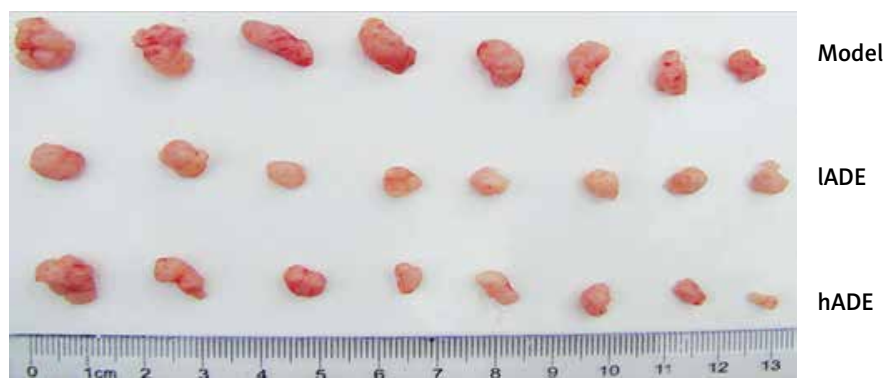

Figure 1. Effect of ADE on tumor growth in vivo. A - Animal weights were quantified once every one week for both Model and ADE treated animals. B - Tumor weight was quantified once every one week. C - Tumor images for Model, hADE, and IADE groups

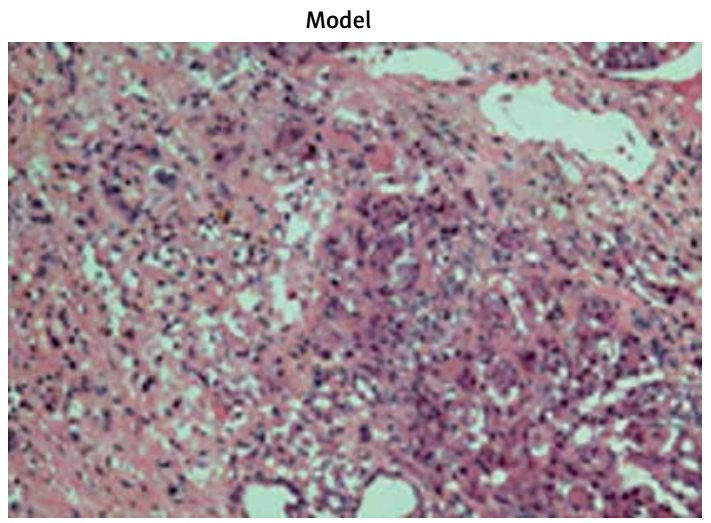

hADE

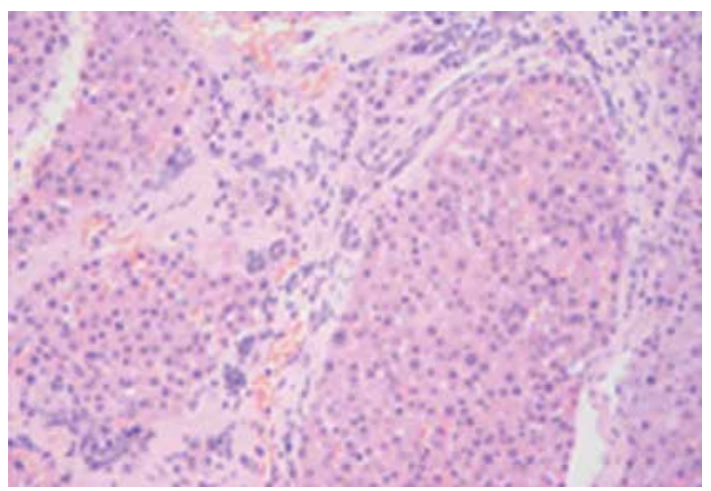

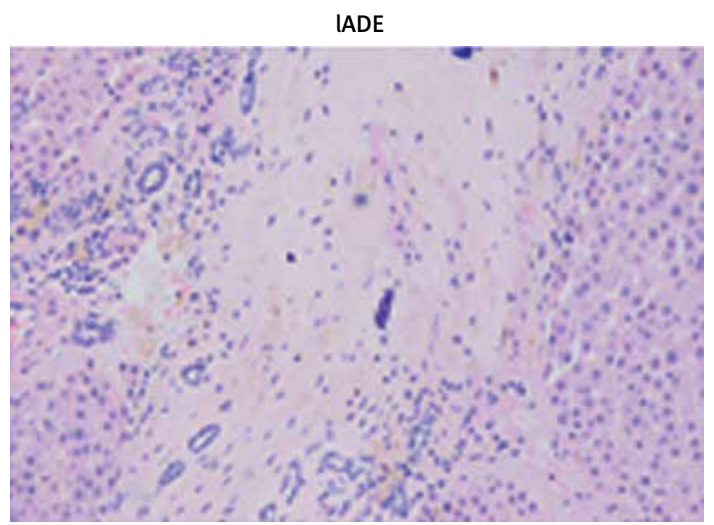

NC

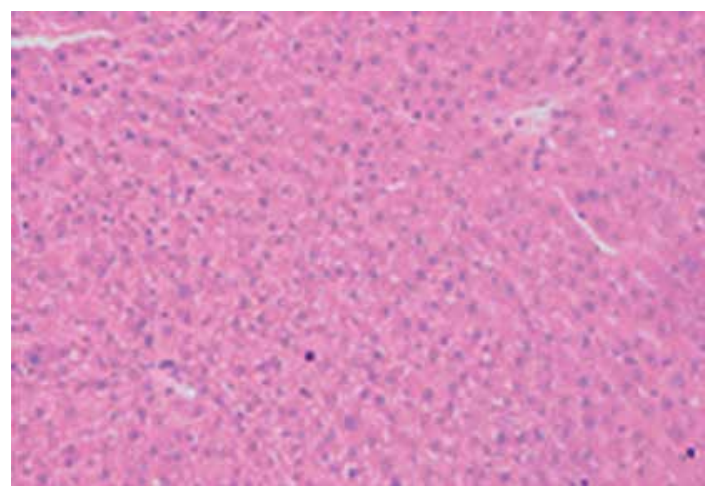

Figure 2. Effect of ADE on pathological development of tumor examined by $\mathrm{IH}$ assay 
co-locational expression of two detected proteins, was clearly observed in the views. We also found that these proteins (SNCG/CD34 and BCRP/CD45,) majorly located around the cellular membrane or in the cytoplasm (Figure $3 \mathrm{~B}$ ). The confocal fluorescence density of double positively-expressed cells in the hADE group $(0.5188 \pm 0.0114$ and 0.6377 \pm 0.0163 , respectively), not the IADE group (0.8113 \pm 0.0523 and $0.7377 \pm 0.1023$, respectively), was statistically reduced compared with the model group ( $p<0.05)$. Meanwhile, all these confocal fluorescence values did not show a significant dif- ference between the hADE group and NC group $(p>0.05)$. WB detection was also performed to determine the expression of SNCG and BCRP. It was found that SNCG and BCRP could be detected in the model group, while the administration of ADE clearly decreased the expression of both proteins in a dose-dependent manner (Figure $3 \mathrm{C}$ ).

\section{Effect of ADE on metastasis}

and proliferation of breast tumor cells

Both proliferation and metastasis are essential properties that display the transitional ability of
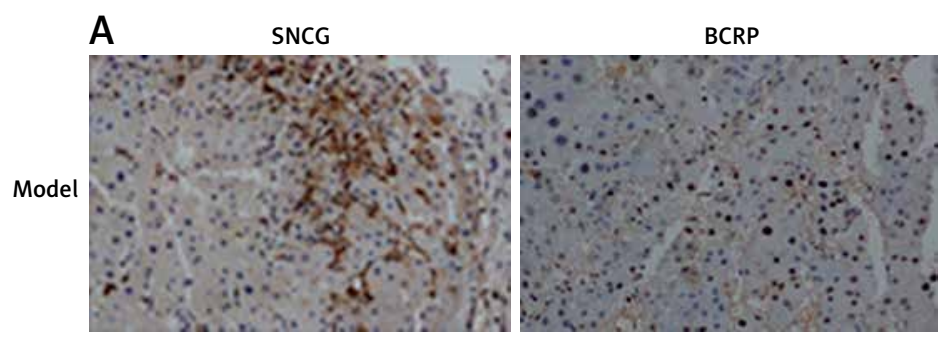

B SNCG

BCRP
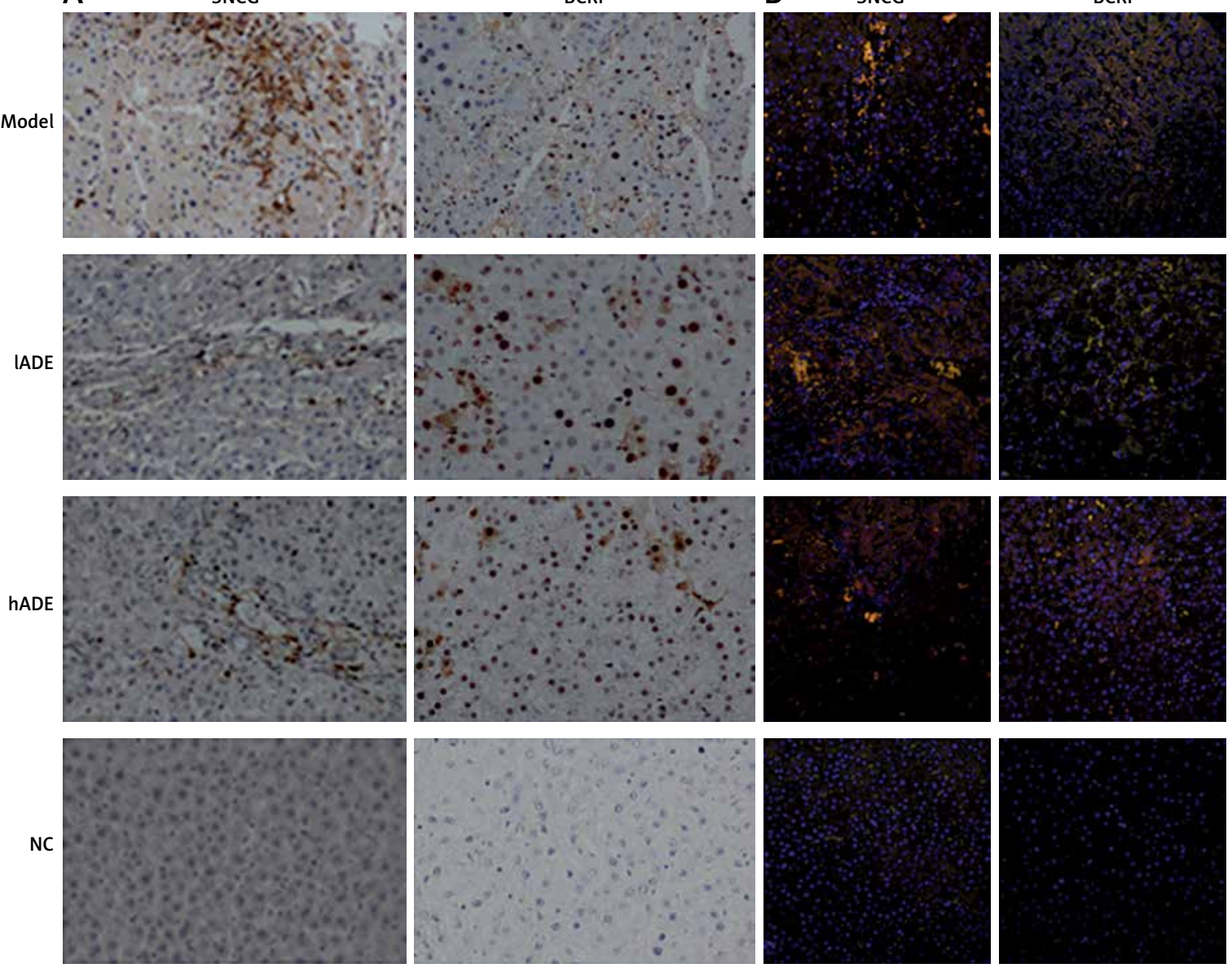

C Model

IADE

hADE

NC
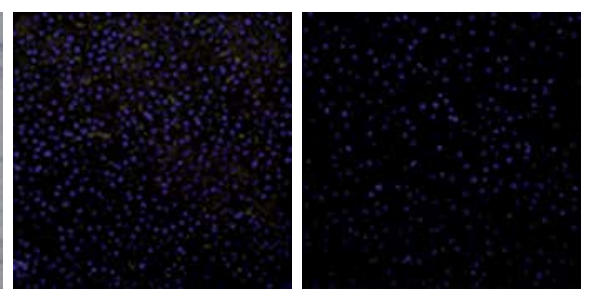

SNCG

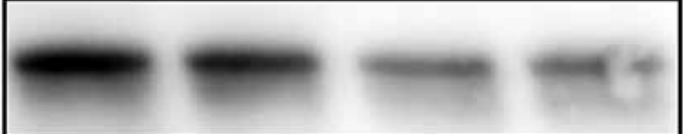

BCRP

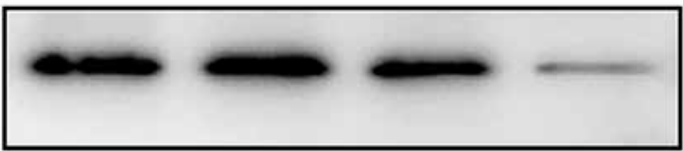

$\beta$-actin

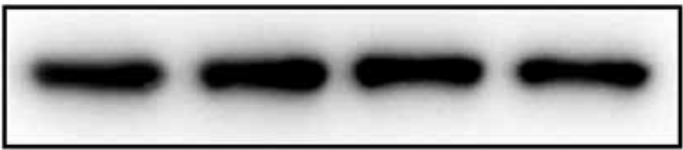

Figure 3. SNCG and BCRP expression in breast tumor. A - Detection of SNCG and BCRP protein for breast tumorigenesis. B - IFA showed the expression of SNCG and BCRP protein in breast tumor cells, and ADE treatment inhibited their expression. C-WB detection showed that SNCG and BCRP protein expression was inhibited by ADE treatment 
cancer cells. Colony formation assay was carried out to detect the effect of ADE on the proliferation rate of MDA-MB-231 cells. We found that administration of $A D E$ resulted in a noticeable increase in colony numbers of MDA-MB-231 cells (Figure 4 A).
The Transwell migration method in vitro was performed to evaluate the effect of ADE on metastasis capacity of MDA-MB-231 cells. The results indicated that $A D E$ decreased the metastasis of MDA-MB-231 cells in a dose-dependent pattern.
A

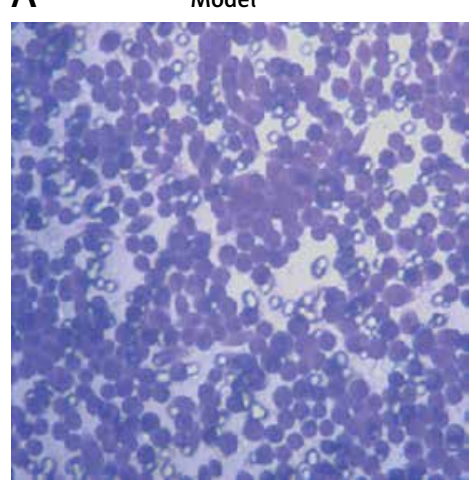

IADE

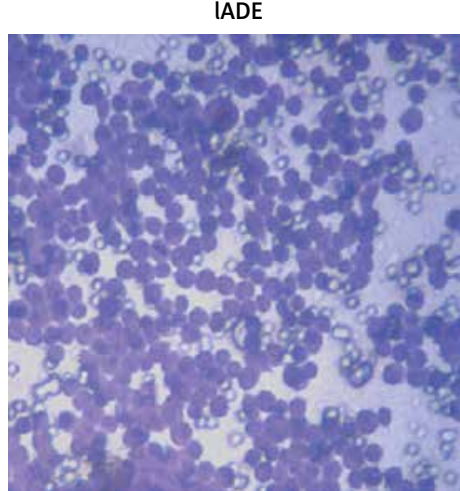

hADE

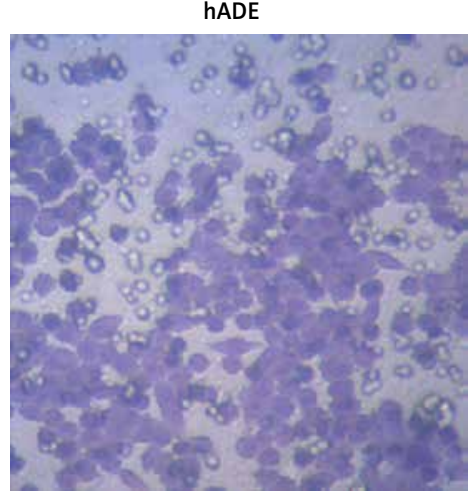

B

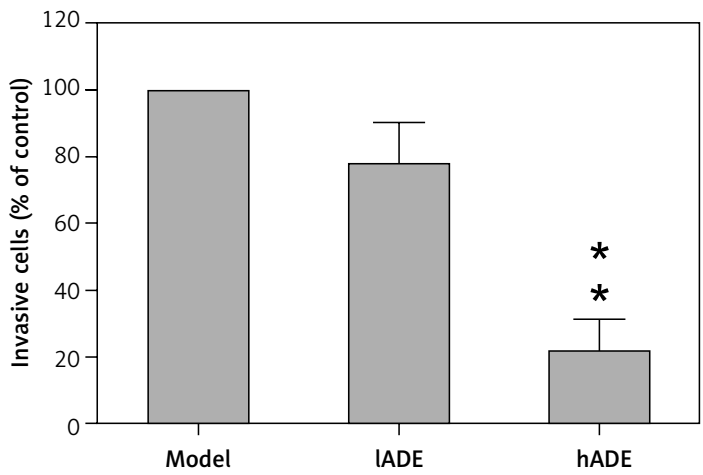

C

Model

IADE

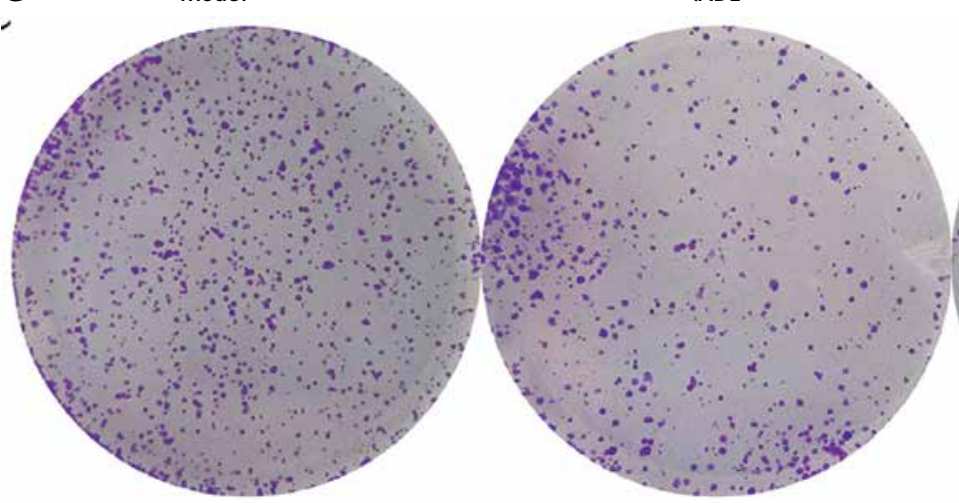

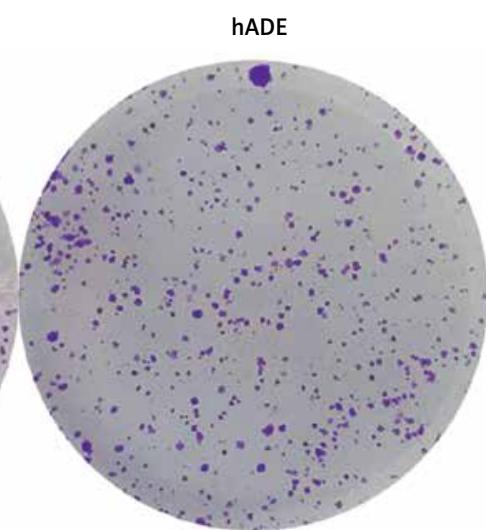

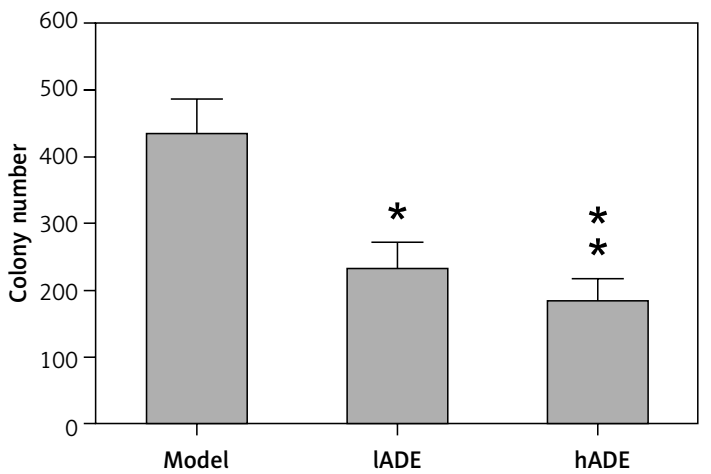

Figure 4. ADE inhibited migration of breast cancer cells. A - Soft agar colony formation assay of the MDA-MB-231 cells with or without treatment of ADE. B - MDA-MB-231 cells were pretreated with 0,25 , and $250 \mu \mathrm{M} A D E$ and were then seeded in the upper wells. After 24 hours, cells on the bottom side of the filter were fixed, stained, and counted. C, D - Colony formation assay was carried out to detect the cell growth of MDA-MB-231 cells after ADE treatment. Values represent the mean \pm SD of three independent experiments performed in triplicate

${ }^{*} p<0.05,{ }^{* *} p<0.01$ compared with control group. 
Cell counting quantification analysis revealed that the invasive efficiency of MDA-MB-231 cells was inhibited by $21.04 \%$ and $78.59 \%$ while cells were administered with 250 and $25 \mathrm{mg} / \mathrm{l}$ ADE at $24 \mathrm{~h}$, respectively (Figure $4 \mathrm{~B}$ ). Furthermore, colony formation assay was also performed to detect the cell growth after ADE administration. The number of generated colonies in ADE treatment groups was significantly lower than in non-treated groups (Figures $4 \mathrm{C}$ and D).

\section{Effect of ADE on EMT process}

The epithelial-mesenchymal transition (EMT) plays an essential role in tumor invasion and metastasis. WB and qRT-PCR were performed to reveal the effect of ADE on the EMT process, through examining the protein and gene expression level of the epithelial marker E-cadherin, mesenchymal marker N-cadherin, vimentin, SNAIL, and additional immuno-suppressor TGF- $\beta 1$.

E-cadherin protein expression pattern in the hADE group $(0.72 \pm 0.04)$ and IADE $(0.42 \pm 0.07)$ were obviously up-regulated compared with the model group $(0.35 \pm 0.03)$ ( $p<0.05)$. In contrast, $\mathrm{N}$-cadherin, vimentin, SNAIL, and TGF- $\beta 1$ protein expression levels in the hADE group $(0.12 \pm 0.08$, $0.59 \pm 0.07,0.11 \pm 0.08$, and $0.32 \pm 0.05)$ were significantly lower compared with those in the model group $(1.00 \pm 0.00)(p<0.05)$ (Figure $5 \mathrm{~A})$. Except for vimentin, all bands of IADE caused less reduction than Hade treatment. In the aspect of mRNA expression, we found that E-cadherin, $\mathrm{N}$-cadherin, SNAIL, and vimentin mRNA expression levels in the ADE groups displayed a significant difference from those in the model group $(p<0.05)$ whereas TGF- $\beta 1$ did not. E-cadherin

A
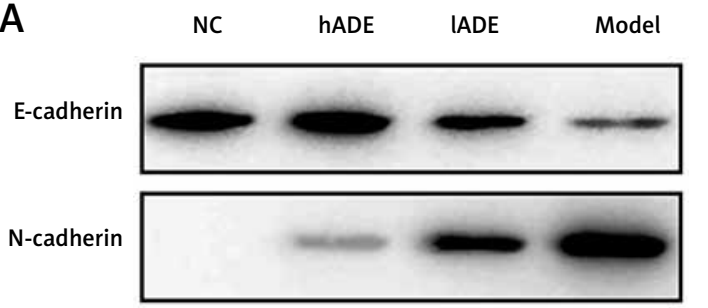

Vimentin

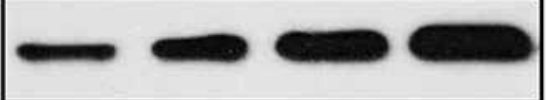

SNAIL

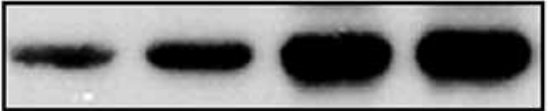

TGF- $\beta 1$

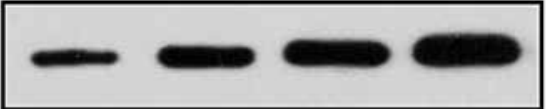

$\beta$-actin

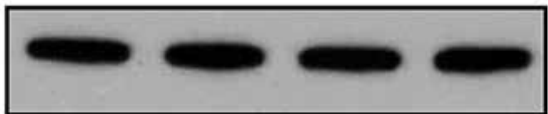

increased while vimentin decreased in the ADE groups (Figure $5 \mathrm{~B}$ ).

\section{Effect of ADE on MAPK and JAK/STAT signaling pathways}

Irregular activation of MAPK and JAK/STAT transduction pathways was closely related to tumorigenesis, the malignant progress and migration; therefore, we performed WB and qRT-PCR to examine protein and gene expression of Raf-1, Mek1, Erk1, and VEGF in the MAPK pathway and JAK2, STAT3, and STAT5 in the JAK/STAT pathway.

Regarding the JAK/STAT pathway, WB data showed that JAK2, STAT3, and STAT5, as well as phosphorylation of STAT3 and STAT5, were significantly down-regulated after hADE and IADE treatment, while these proteins in the model group were up-regulated (Figure 6 A). The qRT-PCR data also showed that JAK2, STAT3, and STAT5 mRNA expression in hADE $(1.95 \pm 0.05,1.85 \pm 0.03$, and $2.15 \pm 0.09$, respectively) and IADE (2.25 \pm 0.04 , $1.92 \pm 0.02$, and $3.85 \pm 0.10$, respectively) groups was significantly lower than in the model group $(5.20 \pm 0.04,3.27 \pm 0.09$, and $5.78 \pm 0.04$, respectively) $(p<0.05)$ (Figure 6 B).

The WB bands showed that the protein expression levels of Raf-1, Mek1, Erk1, and VEGF in the model group were significantly activated compared with the NC group $(0.40 \pm 0.05,0.70 \pm 0.03$, $0.55 \pm 0.11$, and $0.59 \pm 0.05)$; the difference between them was statistically significant $(p<0.05)$. The protein expression levels of Raf-1, Mek1, and Erk1, not VEGF, in hADE group were significantly lower than those in the model group $(p<0.05)$. The others showed no statistically significant difference (Figure $6 \mathrm{C}$ ). The qRT-PCR data, being

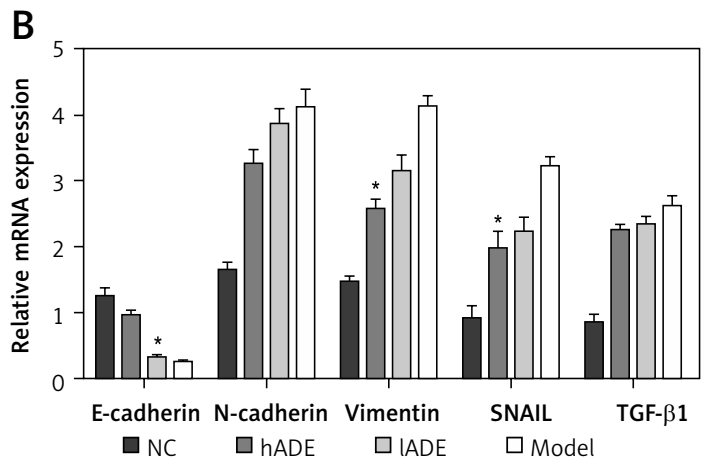

Figure 5. E-cadherin, N-cadherin, vimentin, SNAIL, and TGF- $\beta 1$ expression correlates with breast cancer cells. A - WB analysis of E-cadherin, N-cadherin, vimentin, SNAIL, and TGF- $\beta 1$ protein levels in breast cancer cells. B - qRT-PCR analysis of E-cadherin, N-cadherin, vimentin, SNAIL, and TGF- $\beta 1$ mRNA levels in breast cancer cells 
A

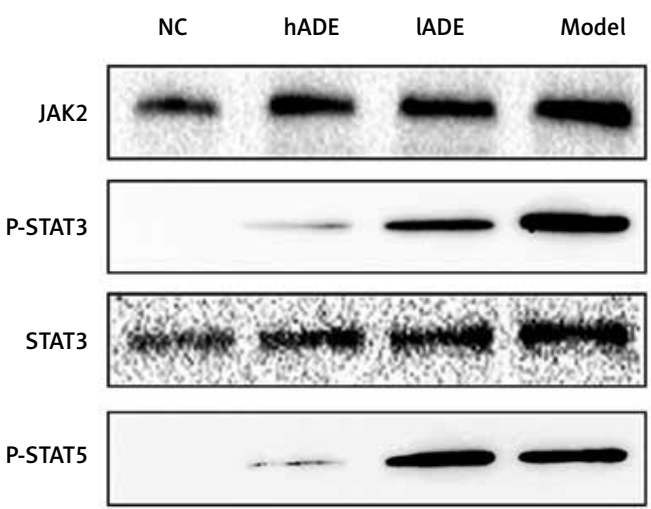

STAT5

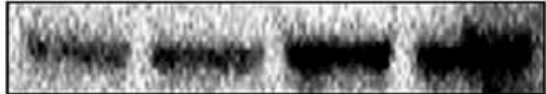

$\beta$-actin

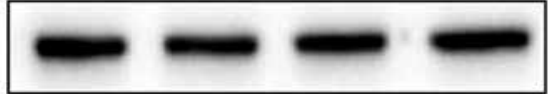

C

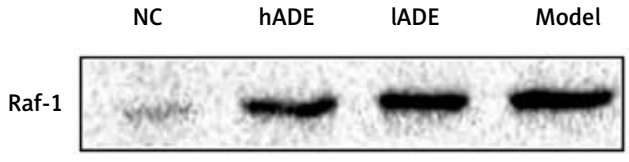

Mek1

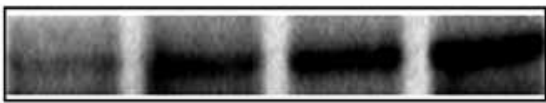

Erk1

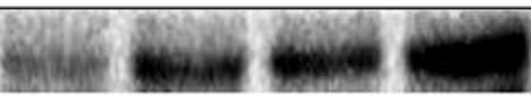

VGEF

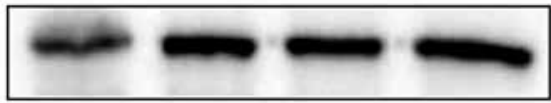

$\beta$-actin

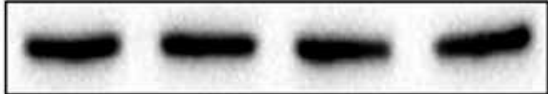

B

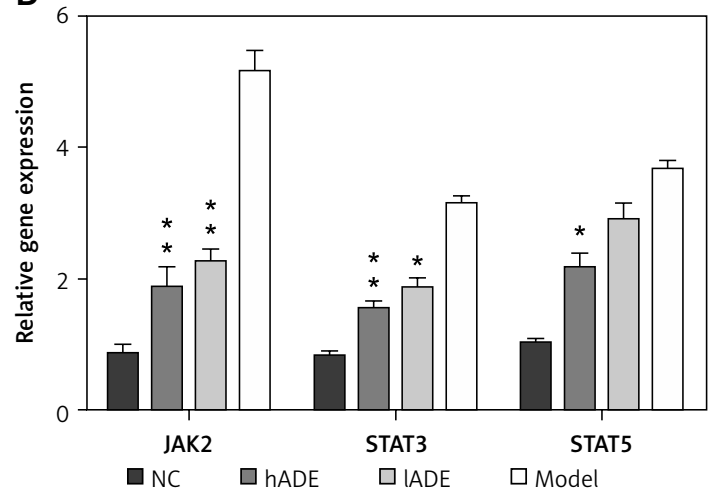

D

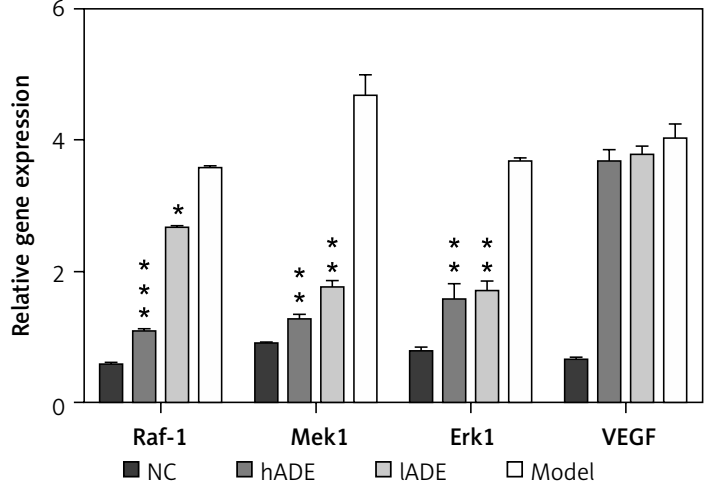

Figure 6. Effect of ADE on MAPK and JAK/STAT signaling pathway activation. A, C - WB analysis of MAPK and JAK/ STAT signaling pathway activation in ADE-treated breast tumor cells; C, D - qRT-PCR analysis of MAPK and JAK/STAT mRNA expression of ADE-treated breast tumor cells at gene level

consistent with the WB data, showed that the mRNA expression levels of Raf-1, Mek1, and Erk1 in hADE $(0.99 \pm 0.02,1.20 \pm 0.05,1.85 \pm 0.09$, and $3.76 \pm 0.24$, respectively) and IADE (1.68 \pm 0.05 , $1.87 \pm 0.06,1.87 \pm 0.11$, and $3.85 \pm 0.20$, respectively) groups were significantly lower compared with those in the model group (with relative density of $3.51 \pm 0.05,4.81 \pm 0.10,3.79 \pm 0.04$, and 4.01 \pm 0.15 , respectively) $(p>0.05)$ (Figure $6 \mathrm{D}$ ).

\section{Discussion}

Breast carcinoma is one of the most common malignant tumors with the characteristics of a low recovery rate and high mortality. CTM possesses a distinct advantage in preventing tumorigenesis, reducing recurrence, and blocking metastasis, and advancing prognosis. The goal of CTM usage is targeting breast tumor cell growth and proliferation.
EMT is a cytological process that enables polarized epithelial cells to transfer into mesenchymal cells and obtain their phenotype, through multiple biochemical and cytological alteration. The changes include abnormal signaling pathway activation, migratory capability, invasiveness, and high resistance to apoptosis [32]. The EMT/MET balance plays an essential role in tumor invasion and metastasis [33-35]. Here we determined the expression of epithelium-specific E-cadherin and mesenchyme-specific $\mathrm{N}$-cadherin, vimentin, TGF- $\beta 1$, and SNAIL via WB and qRT-PCR assays, and found that E-cadherin in the model group was up-regulated and mesenchyme-specific markers decreased, resulting in reduced cell adhesion and obtaining a mesenchymal phenotype, which is considered be a typical phenomenon in tumorigenesis. Additionally, in the ADE group we found that epithelium-specific to mesenchyme-specific alteration was retraced, revealing that ADE treatment facili- 
tated the MET process and consequently brought down the cancer invasion ability.

The MAPK signaling pathway participates in cellular processes, such as replication, differentiation, and apoptosis, through activating cascading signal receptors and transcription factors in the nucleus $[36,37]$. In tumorigenesis, irregular activation of the MAPK signaling pathway could be detected. Activation of the canonical Ras/MAPK pathway is highly infrequent in breast cancer. In the Cancer Genome Atlas study (CGAN, 2012), the frequency of mutations in the Ras and Raf families by next-generation sequencing was less than $2 \%$ of all primary breast tumors [38]. It was also reported that another signal transduction pathway, the JAK/STAT pathway, was found to be exceptionally activated during carcinogenesis [39], and its specific inhibitor, NSC74859, was found to have a suppressive effect on tumorigenesis [40]. STAT3 and STAT5 play important and distinct roles in mammary development and both can be activated in breast cancer. A previous study found that primary human breast tumors displaying activation of both STATs are more differentiated than those with STAT3 activation alone and display more favorable prognostic characteristics [41]. STAT5 and STAT3 mediate opposing effects on several key target genes, with STAT5 exerting a dominant role. Coactivation of STAT5 and STAT3 leads to decreased proliferation and increased sensitivity to the chemotherapeutic drugs paclitaxel and vinorelbine compared with cells that have only STAT3 activation. On the other hand, genetic studies in mouse models support a role for the STAT5 transcription factor as a proto-oncogene in mammary tumor initiation [42]. In the present study, we also found abnormal raised expression of Raf, Mek, and Erk proteins in the modeling process. We also confirmed that JAK/STAT and MAPK signaling pathways were activated in the tumor model group. Notably, the ADE administration effectively inactivated these two pathways, and in consequence reduced breast tumor proliferation and invasion.

Collectively, our data have provided evidence that ADE targeted MAPK and JAK/STAT to suppress EMT and breast tumorigenesis. Recently, ADE has been proved and utilized in anti-inflammatory, wound healing and antiviral remedies [26]; therefore, the clinical employment of CTM as a novel anti-tumor drug might be reasonable and applicable. However, the long-lasting efficacy of ADE to overcome the drug tolerance of tumor cells still need to be further investigated.

\section{Acknowledgments}

This study was supported by the Shandong Science and Technology Research Program (2017GSF218054).

\section{Conflict of interest}

The authors declare no conflict of interest.

\section{References}

1. DeSantis C, Ma J, Bryan L, Jemal A. Breast cancer statistics, 2013. CA Cancer J Clin 2014; 64: 52-62.

2. Koczkodaj P, Sulkowska U, Gotlib J, Mańczuk M. Breast cancer mortality trends in Europe among women in perimenopausal and postmenopausal age (45+). Arch Med Sci 2020; 16: 146.

3. Rethemiotaki I, Rethemiotakis A. Stroke and breast cancer in the United States during 2007-2017. Arch Med Sci Atheroscler Dis 2019; 4: e134-40.

4. Mundy GR. Metastasis to bone: causes, consequences and therapeutic opportunities. Nat Rev Cancer 2002; 2: 584-93.

5. Geng SQ, Alexandrou AT, Li JJ. Breast cancer stem cells: Multiple capacities in tumor metastasis. Cancer Lett 2014; 349: 1-7.

6. Siegel R, Ma J, Zou Z, Jemal A. Cancer statistics, 2014. CA Cancer J Clin 2014; 64: 9-29.

7. Thiery JP, Acloque H, Huang RYJ, Nieto MA. Epithelial-mesenchymal transitions in development and disease. Cell 2009; 139: 871-90.

8. Yilmaz M, Christofori G. EMT, the cytoskeleton, and cancer cell invasion. Cancer Metastasis Rev 2009; 28: 15-33.

9. Son H, Moon A. Epithelial-mesenchymal transition and cell invasion. Toxicol Res 2010; 26: 245-52.

10. Foroni C, Broggini M, Generali D, Damia G. Epithelial-mesenchymal transition and breast cancer: role, molecular mechanisms and clinical impact. Cancer Treat Rev 2012; 38: 689-97.

11. Gonzalez DM, Medici D. Signaling mechanisms of the epithelial-mesenchymal transition. Sci Signal 2014; 7: re8.

12. Xu H, Zhao X, Liu X, Xu P, Zhang K, Lin. Antitumor effects of traditional Chinese medicine targeting the cellular apoptotic pathway. Drug Des Devel Ther 2015; 9: 2735-44.

13. Yao F, Jia R, Huang $H$, et al. Effect of Lactobacillus paracasei N1115 and fructooligosaccharides in nonalcoholic fatty liver disease. Arch Med Sci 2019; 15: 1336-44.

14. Luo KW, Sun JG, Yuet-Wa Chan J, et al. Anticancer effects of imperatorin isolated from Angelica dahurica: induction of apoptosis in HepG2 cells through both death-receptor- and mitochondria-mediated pathways. Chemotherapy 2011; 57: 449-59.

15. Zhao B, Kang Q, Peng Y, et al. Effect of Angelica sinensis root extract on cancer prevention in different stages of an AOM/DSS mouse model. Int J Mol Sci 2017; 18: 1750.

16. Zheng YM, Shen JZ, Wang Y, Xiaoxu Lu A, Ho WS. Anti-oxidant and anti-cancer activities of Angelica dahurica extract via induction of apoptosis in colon cancer cells. Phytomedicine 2016; 23: 1267-74.

17. Kim YK, Kim YS, Ryu SY. Antiproliferative effect of furanocoumarins from the root of Angelica dahurica on cultured human tumor cell lines. Phytother Res 2007; 21: 288-90

18. Houghton J, Stoicov C, Nomura S, et al. Gastric cancer originating from bone marrow-derived cells. Science 2004; 306: 1568-71.

19. Tirode F, Laud-Duval K, Prieur A, Delorme B, Charbord P, Delattre O. Mesenchymal stem cell features of Ewing tumors. Cancer Cell 2007; 11: 421-9.

20. Matushansky I, Hernando E, Socci ND, et al. Derivation of sarcomas from mesenchymal stem cells via inactivation of the Wnt pathway. J Clin Invest 2007; 117: 3248-57. 
21. Mohseny AB, Szuhai K, Romeo S, et al. Osteosarcoma originates from mesenchymal stem cells in consequence of aneuploidization and genomic loss of Cdkn2 J Pathol 2009; 219: 294-305.

22. Tolar J, Nauta AJ, Osborn MJ, et al. Sarcoma derived from cultured mesenchymal stem cells. Stem Cells 2007; 25 371-9.

23. Ren Z, Wang J, Zhu W, et al. Spontaneous transformation of adult mesenchymal stem cells from cynomolgus macaques in vitro. Exp Cell Res 2011; 317: 2950-7.

24. Rubio D, Garcia-Castro J, Martín MC, et al. Spontaneous human adult stem cell transformation. Cancer Res 2005; 65: 3035-9.

25. Wang Y, Huso DL, Harrington J, et al. Outgrowth of a transformed cell population derived from normal human BM mesenchymal stem cell culture. Cytotherapy 2005; 7: 509-19.

26. Ren Z, Zhang YA, Chen Z. Spontaneous transformation of cynomolgus mesenchymal stem cells in vitro: further confirmation by short tandem repeat analysis. Exp Cell Res 2012; 318: 435-40.

27. Fantozzi A, Gruber DC, Pisarsky L, et al. VEGF-mediated angiogenesis links EMT-induced cancer stemness to tumor initiation. Cancer Res 2014; 74: 1566-75.

28. Matysiak M, Kapka-Skrzypczak L, Jodłowska-Jędrych B, Kruszewski M. EMT promoting transcription factors as prognostic markers in human breast cancer. Arch Gynecol Obstet 2017; 295: 817-25.

29. Park AY, Park SJ, Lee J, et al. Simultaneous determination of five coumarins in Angelicae dahuricae Radix by HPLC/UV and LC-ESI-MS/MS. Biomed Chromatogr 2009; 23: 1034-43.

30. Momtazi-Borojeni AA, Nik ME, Jaafari MR, Banach $M$, Sahebkar A. Effects of immunization against PCSK9 in an experimental model of breast cancer. Arch Med Sci 2019; 15: 570-9.

31. Bonnomet A, Syne L, Brysse A, et al. A dynamic in vivo model of epithelial-to-mesenchymal transitions in circulating tumor cells and metastases of breast cancer. Oncogene 2012; 31: 3741-53.

32. Savagner P. The epithelial-mesenchymal transition (EMT) phenomenon. Ann Oncol 2010; 21 Suppl 7: vii89-92.

33. Micalizzi DS, Farabaugh SM, Ford HL. Epithelial-mesenchymal transition in cancer: parallels between normal development and tumor progression. J Mammary Gland Biol Neoplasia 2010; 15: 117-34.

34. Thiery JP. Epithelial-mesenchymal transitions in development and pathologies. Curr Opin Cell Biol 2003; 15: 740-6.

35. Zavadil J, Bottinger EP. TGF-beta and epithelial-to-mesenchymal transitions. Oncogene 2005; 24: 5764-74.

36. Deming D, Geiger P, Chen H, Vaccaro A, Kunnimalaiyaan M, Holen K. ZM336372, a Raf-1 activator, causes suppression of proliferation in a human hepatocellular carcinoma cell line. J Gastrointest Surg 2008; 12: 852-7.

37. O'Neill E, Kolch W. Conferring specificity on the ubiquitous Raf/MEK signalling pathway. Br J Cancer 2004; 90: 283-8.

38. Cerami E, Gao J, Dogrusoz U, et al. The cBio cancer genomics portal: an open platform for exploring multidimensional cancer genomics data. Cancer Discov 2012; 2: 401-4.

39. Calvisi DF, Ladu S, Gorden A, et al. Ubiquitous activation of Ras and Jak/Stat pathways in human HCC. Gastroenterology 2006; 130: 1117-28

40. Hou J, Tang D, Xu Y, He X, Wei J. Effect of janus kinase-signal transducer and activator of transcription 3 inhibitor (Piceatannol) on the growth of hepatocellular carcinoma in vitro. Chin J Exp Surg 2010; 27: 1822-4.

41. Walker SR, Nelson EA, Zou L, et al. Reciprocal effects of STAT5 and STAT3 in breast cancer. Mol Cancer Res 2009; 7: 966-76.

42. Wagner KU, Rui H. Jak2/Stat5 signaling in mammogenesis, breast cancer initiation and progression. J Mammary Gland Biol Neoplasia 2008; 13: 93-103. 